\title{
Tumor del cuerpo carotídeo: A propósito de 10 casos tratados
}

\author{
Sebastián Soto $\mathrm{G}^{1,3}$, Francisco Valdés $\mathrm{E}^{1,3}$, \\ Albrecht Krämer $\operatorname{Sch}^{1,3}$, Leopoldo Mariné $M^{1,3}$, Michel \\ Bergoeing $R^{1,3}$, Renato Mertens $M^{1,3}$, Antonieta Solar $\mathbf{G}^{2}$, \\ Annerleim Walton $D^{3}$, Jeannette Vergara $G^{1,3 a}$. \\ Carotid body tumors: \\ Report of ten cases
}

Background: Carotid body tumors arise from a cellular conglomerate located at the carotid bifurcation. Progressive enlargement can involve the arterial wall and neighbor cranial nerves. Aim: To report a series of 10 patients treated of carotid body tumors and review national experience. Patients and methods: Between 1984 and 2006, we operated 8 women and 2 men, aged 19 to 75 years, with this type of tumor. Results: The most common cause for consultation was a cervical mass in $90 \%$, with a mean evolution lapse of 13.2 months (range 3 to 126). In all cases, diagnosis was confirmed with angiographic imaging and histopathology. Ten tumors were surgically removed with no complications. Eighty percent of tumors were in stage II according to Shamblin classification. During long term follow up all patients have remained asymptomatic. Only 31 carotid body tumors have been reported in Chilean medical literature during a 43 year period. Conclusions: Paragangliomas of the carotid body can be diagnosed in clinical grounds, requiring vascular imaging. These infrequent lesions are generally benign, early surgical removal by surgeons with vascular expertise avoids neurological and or vascular complications (Rev Méd Chile 2007; 135: 1414-20).

(Key words: Angiography; Carotid body tumor; Paraganglioma, exta-adrenal)

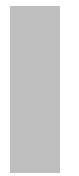

Recibido el 12 de abril, 2007. Aceptado el 28 de junio, 2007.

Sección de Cirugía Vascular y Endovascular. Departamentos de Enfermedades Cardiovasculares $^{1}$ y Anatomía Patológica ${ }^{2}$. División de Cirugía ${ }^{3}$, Facultad de Medicina y Hospital Clínico Pontificia Universidad Católica de Chile. Santiago de Chile.

${ }^{a}$ Enfermera Universitaria

$\mathrm{L}$ os tumores del cuerpo carotídeo son neoplasias poco frecuentes en nuestro medio, que se originan en la estructura del mismo nombre ubicada a nivel de la bifurcación carotídea ${ }^{1-4}$.

Correspondencia a: Dr. Francisco Valdés E. Apoquindo 3990, Of. 601, Las Condes, Santiago de Chile. Teléfono: 562-2070721. Fax: 56-2-2070718. E mail: franval@med.puc.cl
Dada la localización estratégica de este tumor en el triángulo carotídeo, su crecimiento, aunque lento, resulta evidente al paciente. Su desarrollo puede llegar a envolver la carótida común y su bifurcación, así como uno o más nervios craneanos de la vecindad. Por tal motivo, es recomendable su extirpación.

El tratamiento de elección es la cirugía, cuyas primeras descripciones datan del siglo XVIII, 
siendo Scudder, en 1903, quien realizó la primera resección exitosa preservando el sistema carotí$\mathrm{deo}^{2,3}$. En 1940, Gordon-Taylor describió la disección subadventicial, la cual es ampliamente aceptada en la actualidad, habiendo sido reportados más de mil casos operados hasta fines del siglo pasado en todo el planeta ${ }^{1-4}$.

Según el tamaño, extensión e invasión de las estructuras vecinas, la extirpación del tumor puede derivar en serias complicaciones, por lo que Shamblin ${ }^{5}$ propuso la siguiente clasificación:

Tipo I: Sin compromiso de estructuras vecinas (fácilmente disecable). Tipo II: Compromiso adherencial a la adventicia, crecimiento circunferencial en la bifurcación carotídea, desplazamiento de pares craneanos y extensión hacia la base del cráneo (más frecuente, requiere resección subadventicial). Tipo III: Marcado compromiso envolvente de la bifurcación carotídea y los pares craneanos, desplazamiento de las estructuras vecinas (mayor incidencia de complicaciones neurológicas, puede requerir resección en bloque y reconstrucción vascular con técnicas de preservación cerebral).

En la presente comunicación analizamos nuestra experiencia con 10 pacientes tratados.

\section{Material y MÉTODO}

Se evalúa retrospectivamente nuestra experiencia en el manejo de 10 pacientes portadores de paragangliomas del cuerpo carotídeo, tratados entre enero de 1984 y diciembre de 2006.

Se analiza la edad, el sexo, el tamaño y la extensión del tumor (según Shamblin), el tipo de cirugía efectuada, el tiempo operatorio, las complicaciones intraoperatorias y posoperatorias inmediatas, histopatología y resultados alejados. Se construyó una base de datos utilizando el programa Microsoft Excel y el programa "STATA 9".

Se revisó exhaustivamente la literatura médica nacional, incluyendo la Revista Médica de Chile, la Revista Chilena de Cirugía, los Archivos de la Sociedad de Cirujanos de Chile y la Revista Chilena de Otorrinolaringología y Cirugía de Cabeza y Cuello.

\section{Resultados}

Durante el período del estudio se trataron 10 pacientes portadores de 11 tumores de cuerpo carotídeo, 8 de ellos de sexo femenino y 2 de sexo masculino, con una relación mujer:hombre de 4:1. La edad de la serie fluctuó entre 19 y 75 años, con una media de 56 años (Tabla 1).

El motivo de consulta más frecuente fue un aumento de volumen cervical en la zona del triángulo carotídeo ( $\mathrm{n}=9$ ). El tiempo de evolución medio fue 13,2 meses, fluctuando entre 3 y 126 meses. En 6 casos, al momento del diagnóstico la única alteración fue el aumento de volumen cervical. Cuatro pacientes referían: tinitus, parestesia facial ipsilateral y disminución de agudeza visual y un caso refirió anorexia y baja de peso. En un caso se auscultó un soplo cervical. En un caso fue un hallazgo ecográfico.

Se utilizaron diversos métodos de diagnóstico por imágenes, siendo la angiografía convencional el más utilizado (60\%). Su uso ha disminuido desde la aparición de la angiografía por tomografía computada y la resonancia magnética.

En 8 pacientes el tumor afectó la bifurcación carotídea derecha, con un caso bilateral. En una paciente el tumor fue detectado durante el curso del embarazo, siendo observado hasta completar la gestación y resecado en forma diferida, sin inconvenientes.

El tiempo operatorio fluctuó entre 120 y 180 min, con una media de $147 \mathrm{~min}$. En todos los casos se identificaron y aislaron los nervios craneanos en relación al tumor, incluso aquellos incluidos en su masa, sin lesionarlos (Figuras 1A y 1B). Durante el posoperatorio inmediato y alejado, ningún paciente presentó déficit neurológico central ni periférico.

La hospitalización media fue de 4 días, con rango entre 3 y 7 días.

De los 10 tumores resecados, 8 correspondían al tipo II de la clasificación de Shamblin y sólo requirieron extirpación. En los 2 casos restantes, el tumor correspondió al tipo III. Uno se manejó con resección del tumor en bloque, ligadura de la arteria carótida externa y reconstrucción arterial mediante interposición carótido-carotídea de un segmento de vena safena bajo protección cerebral con shunt, y en el otro caso se efectuó resección con reanastomosis carotídea término-terminal con igual método de protección cerebral. 
Tabla 1. D atos demográficos y resultados en 10 pacientes operados por tumor del cuerpo carotídeo

\begin{tabular}{|c|c|c|c|c|c|c|c|c|c|c|}
\hline Caso & Sexo & $\begin{array}{l}\text { Edad } \\
\text { (años) }\end{array}$ & $\begin{array}{l}\text { Patologías } \\
\text { asociadas }\end{array}$ & Estudio & Ubicación & $\begin{array}{l}\text { Tamaño } \\
(\mathrm{cm})\end{array}$ & Cirugía efectuada & Complic. & $\begin{array}{l}\text { Año } \\
\text { Tto }\end{array}$ & $\begin{array}{c}\text { Tipo } \\
(*)\end{array}$ \\
\hline 1 & $\mathrm{~F}$ & 46 & Hipertiroidismo & Angio & Derecho & $3 \times 4$ & Resección & No & 1984 & 2 \\
\hline 2 & M & 19 & No & Angio & Derecho & $6 \times 8$ & $\begin{array}{l}\text { Resección en } \\
\text { block. Puente con } \\
\text { safena de carótida } \\
\text { común a la } \\
\text { interna }\end{array}$ & No & 1984 & 3 \\
\hline 3 & $\mathrm{~F}$ & 68 & No & Angio, TAC & Derecho & $5 \times 3$ & Resección & No & 1995 & 2 \\
\hline 4 & M & 58 & No & TAC, RNM & Derecho & $6,4 \times 4,5$ & Resección & No & 1997 & 2 \\
\hline 5 & $\mathrm{~F}$ & 75 & No & Eco, Angio & Derecho & $6 \times 4$ & Resección & No & 1997 & 2 \\
\hline 6 & $\mathrm{~F}$ & 73 & No & TAC, Angio & Derecho & $9 \times 7$ & $\begin{array}{l}\text { Resección } \\
\text { Re- anastomosis } \\
\text { carotidea }\end{array}$ & No & 1997 & 3 \\
\hline 7 & $\mathrm{~F}$ & 33 & $\begin{array}{l}\text { No (diagnóstico } \\
\text { durante } \\
\text { gestación) }\end{array}$ & $\begin{array}{l}\text { EcO, } \\
\text { TAC }\end{array}$ & Derecho & $6 \times 4$ & Resección & No & 2003 & 2 \\
\hline 8 & $\mathrm{~F}$ & 70 & $\begin{array}{l}\text { Hipertensión } \\
\text { arterial }\end{array}$ & Angio & Derecho & $6 \times 4$ & Resección & No & 2003 & 2 \\
\hline 9 & $\mathrm{~F}$ & 46 & No & Eco, RNM & $\begin{array}{l}\text { Derecho } \\
\text { (Tu Izquierdo } \\
\text { en control) }\end{array}$ & $5 \times 3$ & Resección & No & 2004 & 2 \\
\hline 10 & $\mathrm{~F}$ & 37 & No & Eco, TAC & Derecho & $4 \times 3$ & Resección & No & 2006 & 2 \\
\hline
\end{tabular}

*Tipo según Shamblin. Eco =Ecografía Doppler color. RNM: Resonancia nuclear magnética. TAC: Tomografía axial computada.

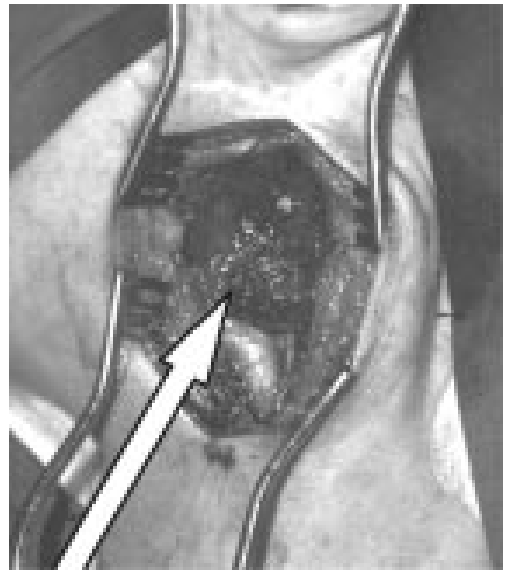

A. Paraganglioma in situ (flecha).

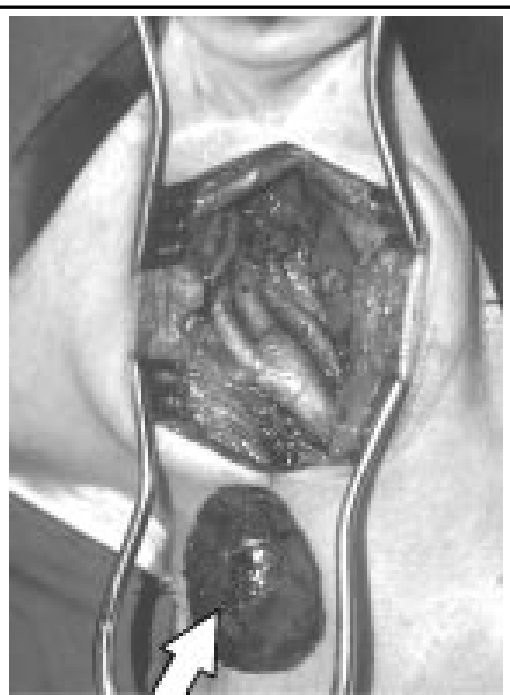

B. Tumor ya resecado (flecha).

Figura 1. Vista operatoria pre y post resección de glomus carotídeo. 
En todos los casos, la histopatología confirmó el diagnóstico (Figuras 2A, 2B y 2C ).

Los resultados de la búsqueda efectuada en la literatura médica chilena se resumen en la Tabla 2.

\section{DisCUSIÓN}

Este tumor se origina en el cuerpo carotídeo, conglomerado celular derivado del mesodermo del tercer arco branquial ${ }^{2}$, conocido como paraganglioma (nido de células neuroendocrinas procedentes de la cresta neural). Varía en tamaño entre 2 y $5 \mathrm{~mm}$. Se ubica en la zona media de la adventicia y en ocasiones periadventicial ${ }^{4}$, siendo inervado por el nervio glosofaríngeo e irrigado por ramas de la arteria carótida externa ${ }^{1,4}$.

Histológicamente, el tumor se organiza en nidos de estructura alveolar conocidos como Zellbalen, los que se componen de 3 tipos celulares que participan en la regulación cardiorrespiratoria, siendo altamente sensibles a las variaciones del $\mathrm{pH}$ sanguíneo, anhídrido carbónico y presión parcial de oxígeno ${ }^{1}$. Los Zellbalen están compuestos por células tipo I productoras de catecolaminas y una amplia variedad de péptidos inmunorreactivos, células tipo II o sustentaculares que rodean a las anteriores, teniendo características similares a las células de Schwann y tipo III que corresponden a terminales nerviosos sensitivos que se originan del ganglio sensitivo del nervio glosofaríngeo 5 .

La respuesta celular a las variaciones de la $\mathrm{PaO}_{2}$ es amplia: desde la atrofia frente a hiperoxia sostenida, hasta la hipertrofia e hiperplasia frente a la hipoxia sostenida o intermitente ${ }^{6}$.

La aparición de paragangliomas del cuerpo carotídeo puede ser esporádica o familiar, siendo el primer tipo el más frecuente, generalmente unilateral y asociado en forma directa a la hipoxia crónica $^{1,2,4,7}$.

Desde las primeras descripciones del paraganglioma del cuerpo carotídeo en el siglo XVIII hasta la actualidad, su incidencia ha sido escasa, lo que se refleja en un total cercano a los 1.000 casos reportados hasta fines del siglo $\mathrm{XX}^{2,4}$. En nuestro medio, la frecuencia del tumor del cuerpo carotídeo es baja; de hecho, desde la primera comunicación en $1963^{8}$ a la fecha, sólo existen publicaciones de casos aislados o series pequeñas, que incluida la presente, suman sólo 31 lesiones ${ }^{8-16}$. (Tabla 2). Es posible que haya más casos tratados y no reportados, sin embargo es improbable que sea un número significativo.

En poblaciones residentes en alturas superiores a los 4.000 metros sobre el nivel del mar, la incidencia es significativamente mayor. En un periodo de 35 años, en un estudio multicéntrico
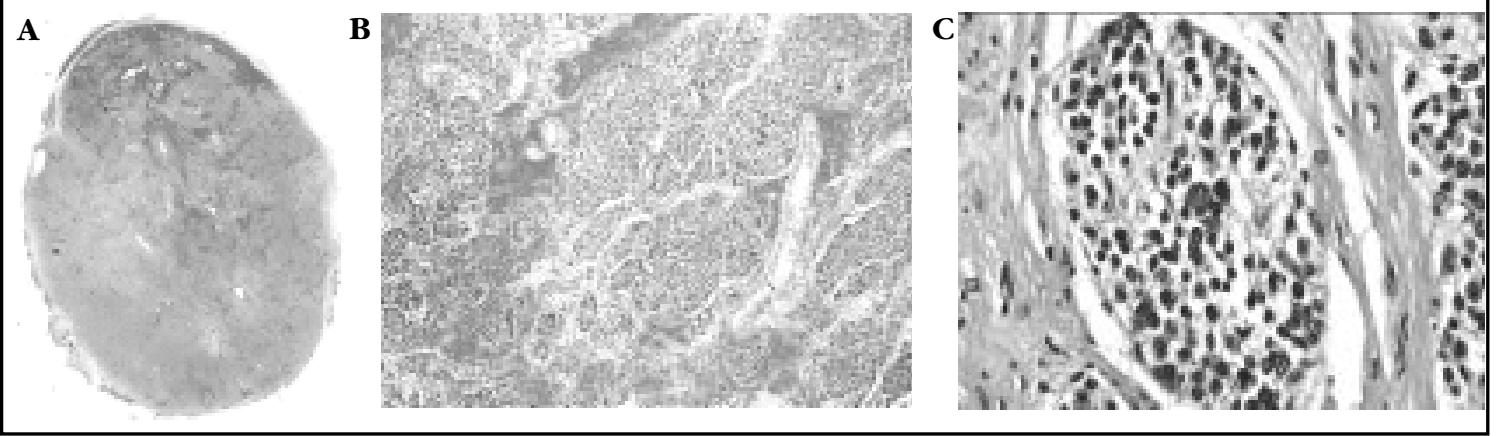

Figura 2 A, B y C. Histopatología de glomus carotídeo.

A. Corte transversal de un tumor del cuerpo carotídeo. Tumor de arquitectura algo lobular, con zonas de hemorragia, rodeado por una cápsula delgada y fibrosa (HE, vista con lupa, x 20).

B. Area con hemorragia e hiperemia marcadas, que separan los grupos de células tumorales y destacan la disposición de éstas en nidos (Zellballen) (HE, aumento mediano, x 200).

C. Nido de células tumorales de citoplasma rosado y granular y núcleos redondos, pequeños y uniformes. Al centro se observan núcleos pleomórficos y en la periferia destacan las células sustentaculares de núcleos lentiformes (HE, aumento mayor, x 400). 
Tabla 2. Tumores del cuerpo carotídeo en la literatura médica chilena

\begin{tabular}{|c|c|c|c|c|c|c|c|c|c|c|}
\hline $\begin{array}{l}\# \\
\text { Ref }\end{array}$ & Autor & Año & $\mathrm{N}$ & $\begin{array}{l}\text { Sexo } \\
M / F\end{array}$ & Doppler & Angio & $\mathrm{CT} / \mathrm{RM}$ & Reseccion & \multicolumn{2}{|c|}{$\begin{array}{l}\text { Morbilidad } \\
\text { N. Craneal/Central/Vasc }\end{array}$} \\
\hline \multirow[t]{2}{*}{8,13} & Contreras & 1963 & & & & & & & & \\
\hline & & 1990 & 5 & $4 / 1$ & - & & & 5 & $0 / 1 / 1$ & \\
\hline \multirow[t]{2}{*}{9} & Morán & 1969 & 1 & 1 & - & - & - & 1 & 0 & \\
\hline & González, & 1975 & & & & & & & & \\
\hline 10, & Volpato, & 1982 & & & & & & & & \\
\hline 11,14 & Torres & 1990 & 9 & $4 / 5$ & - & 5 & 2 & 7 & $5 / 3 / 3$ & \\
\hline 1 & Valdés & 1985 & 1 & $0 / 1$ & - & 1 & - & 1 & 0 & \\
\hline 12 & Pérez & 1988 & 2 & $1 / 1$ & - & 1 & - & 2 & $1 / 0 / 0$ & \\
\hline 15 & Marín & 1996 & 1 & $1 / 0$ & - & 1 & 1 & 1 & 0 & Reconstrucción arterial \\
\hline \multirow[t]{3}{*}{16} & Nazar & 2005 & 2 & $0 / 2$ & 1 & 1 & 2 & 2 & $1 / 0 / 0$ & 1 Embolización preoperatoria \\
\hline & Soto* *** & 2007 & 11 & $2 / 8$ & 4 & 6 & 7 & 10 & 0 & 2 Casos: reconstrucción arterial \\
\hline & Total & & 31 & $12 / 19$ & & & & 28 & & \\
\hline
\end{tabular}

* 1 caso bilateral operado 1 lado. ** Serie incluye caso de ref\#1.

boliviano, se reportó un total de 323 casos, 80\% mujeres, todos provenientes de las ciudades de La Paz, Oruro y Potosí (observaciones no publicadas).

La base para el diagnóstico se fundamenta en la sospecha y el examen clínico. Se trata de una masa submandibular indolora, de crecimiento lento, móvil en sentido lateral, pero a su vez fija en sentido longitudinal ${ }^{1-5}$, característica semiológicamente conocida como signo de Fontaine. El crecimiento lento y gradual se traduce en una demora entre su aparición y la consulta ${ }^{9}$, tal como ocurrió en la presente serie (tiempo medio entre aparición o síntomas y consulta 13,2 meses).

Según algunas publicaciones, no existiría diferencia entre sexos, con edad de presentación variable, generalmente en la edad adulta entre 25 y 75 años ${ }^{1-3,17}$. Sin embargo, en nuestra casuística, $80 \%$ de los casos son de sexo femenino, al igual como ocurre en Bolivia.

Existe una tendencia familiar entre $10 \%$ y $35 \%$ de los casos en las distintas series. Se trata de tumores bilaterales, asociados al síndrome de neoplasia endocrina múltiple tipo II, con herencia ligada al sexo masculino $2,3,17$.

Los tumores malignos representan entre 5\%-7\% de los casos, se presentan a edades más tempranas, se manifiestan mediante invasión locorregional y, en algunos casos, con metástasis hepáticas y pulmonares. El screening a todos los casos no se justifica por el bajo porcentaje de malignidad. Sí debe realizarse en casos en que el estudio histopatológico revele malignidad $4,6,8,17-19$.

En algunos casos existe asociación con otros tumores, tal como el paraganglioma del glomus timpánico o yugular, lo que obliga a un cuidadoso examen de oído, nariz y orofaringe previo a la cirugía, lesiones que generalmente resultan aparentes en el estudio imagenológico ${ }^{17-19}$.

Algunas comunicaciones actuales mencionan la biopsia por punción como herramienta diagnóstica ${ }^{17-19}$. En nuestra opinión, ésta se encuentra contraindicada por el alto riesgo de hemorragia que presenta este tumor intensamente vascularizado, evento que dificulta el tratamiento posterior e incluso puede ocasionar la muerte del paciente 3,8 .

Algunos autores sugieren la angiorresonancia nuclear magnética como el gold standard de los estudios imagenológicos $2,3,17-20$. Sin embargo, dicho estudio tiene un alto costo comparado con las nuevas tecnologías en base a ecografía y tomografía axial computada. Las imágenes obtenidas con estos métodos cumplen los requisitos necesarios para el screening y la planificación de una eventual cirugía, entregando información topográfica y funcional satisfactorias ${ }^{2,3,8,21,22}(\mathrm{Fi}-$ guras 3,4 y 5 ). 


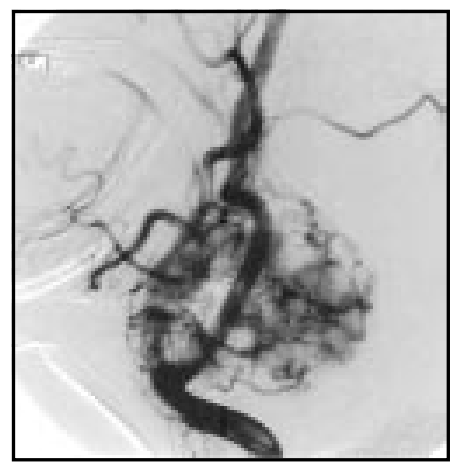

Figura 3. Angiografía carotídea selectiva, que evidencia masa hipervascularizada en la bifurcación carotídea derecha.

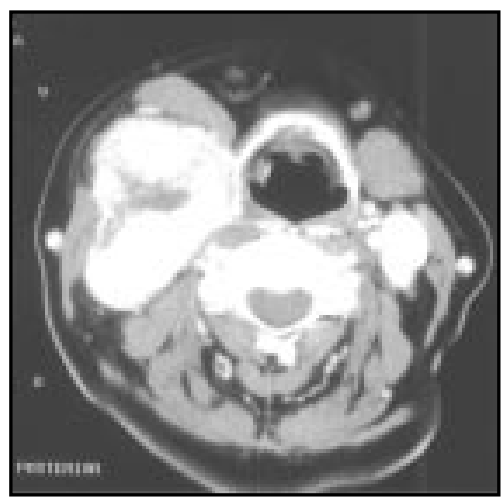

Figura 4. Angio-escáner del cuello (corte transversal), que evidencia gran tumor hipervascularizado en relación a bifurcación carotídea derecha que envuelve ambas ramas de la carótida.

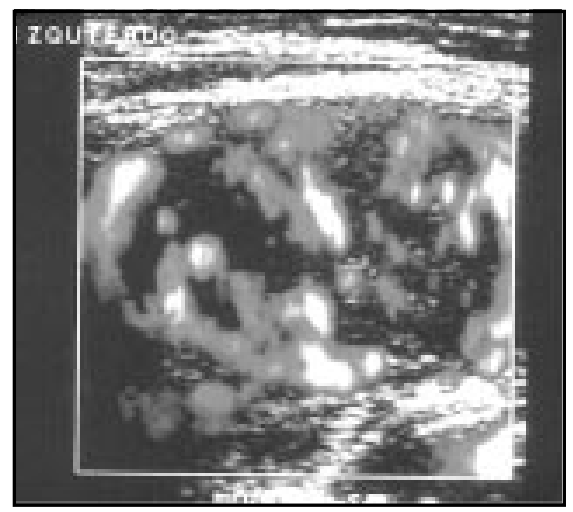

Figura 5. Eco duplex que muestra gran masa vascularizada a nivel del bulbo carotídeo izquierdo, en estrecha relación con la bifurcación carotídea.
Como diagnóstico diferencial se debe tener presente todos los tumores laterales del cuello: el quiste branquial, el tumor mixto de la parótida y otros tumores de glándulas salivales, el aneurisma carotídeo, las adenopatías cervicales benignas o malignas, al neurofibroma, y el hemangioma ${ }^{1}$.

El tratamiento de elección continúa siendo la resección quirúrgica, tanto en los casos benignos como malignos, incluyendo las metástasis a distancia, las cuales presentan nula respuesta ante la radio o quimioterapia ${ }^{1-3,20}$.

Poseedor de un genio biológico benigno en 90\%-95\% de los casos $^{2}$, su extirpación es un desafío para el cirujano, dada la compleja anatomía de la zona involucrada y de las estructuras nobles adyacentes que se ven distorsionadas y, a veces, completamente rodeadas por el crecimiento del tumor. La cirugía de tumores avanzados (clasificación de Shamblin II, y III) se asocia a mayor morbilidad por trauma de nervios craneanos o isquemia cerebral, como demostró Hallet en un estudio de la Clínica Mayo, que incluyó 153 pacientes consecutivos $^{22}$.

Algunos autores preconizan la embolización selectiva previa a la resección quirúrgica, lo que permitiría un menor sangrado intraoperatorio y una mejor diferenciación de las distintas estructuras comprometidas ${ }^{23}$. Sin embargo, esta técnica no está exenta de complicaciones por compromiso isquémico central e incluso muerte ${ }^{24-25}$.

\section{Conclusión}

El tumor o paraganglioma del cuerpo carotídeo es una patología infrecuente en Chile, cuyo estudio implica una evaluación angiográfica. La resección debe ser efectuada por un cirujano que domine las técnicas de reconstrucción vascular del territorio carotídeo, en particular en los casos de mayor tamaño (Shamblin II y III), con el objetivo de minimizar las complicaciones neurológicas centrales y periféricas. 


\section{REFERENCIAS}

1. Valdés F, Rosenberg H, Krämer A, Huete I. Tumor del Cuerpo Carotídeo. Rev Méd Chile 1985; 113: 1108-12.

2. Knight tT Jr, González Ja, Rary JM, Rush DS. Current concepts for the surgical management of carotid body tumor. Am J Surg 2006; 191: 104-10.

3. Sorba A, Nikeghbalian S, Yarmohammadi H, AssadiSABET A. Surgical management of carotid body tumors: A 24-year Surgical Experience. ANZ J Surg 2006; 76: 214-7.

4. Maxwell JG, Jones SW, Wilson E, Kotwall CA, Hall T, Hamann S, Brinker CC. Carotid body tumor excisions: adverse outcomes of adding carotid endarterectomy. J Am Coll Surg 2004; 198: 36-41.

5. Shamblin WR, Remine WH, Sheps SG, Harrison EG JR. Carotid Body Tumor (Chemodectoma): Clinicopathologic analysis of ninety cases. Am J Surg 1971; 122: 732.

6. Kummer W, Yамамото Y. Cellular distribution of oxygen sensor candidates-oxidases, cytochromes, $\mathrm{K}+$ - channels-in the carotid body. Microsc Res Tech 2002; 59: 234-42.

7. Lahiri S, Di Giulio C, Roy A. Lessons from chronic intermittent and sustained hypoxia at high altitudes. Res Phys Neurobiol 2002; 130: 223-33.

8. Contreras O, Jeon J, Torres J, Cea A. Tumores del Cuello. Arch Soc Cir Chile 1963; 15: 461-5.

9. Morán S, Passi J, Etchart M, Vásquez A. Tumor del cuerpo carotídeo (quemodectoma). Comunicación de 1 caso y revisión de la literatura. Arch Soc Cir Chile 1969; 21: 127-9.

10. González M. Tumores primitivos del cuello. Arch Soc Cir Chile 1975; 27: 323-32.

11. Volpato R, Torres J, Power E. Tumores primitivos del cuello. Experiencia clínica. Rev Chil Cir 1982; 34: 394-7.

12. Pérez Ja, Norambuena L, Valencia V, Iribarren O, Kinast C, Jiménez L. Tumores del cuerpo carotídeo. Casos Clínicos. Rev Chil Cir 1988; 4: 260-2.

13. Contreras O, González M, Irarrázabal V, Villalobo R, HäBrerle S. Paragangliomas en cabeza y cuello. Rev Chil Cir 1990; 42: 136-9.

14. Torres J, Volpato R, Power E, Martínez V, Jorquera G. Paragangliomas de la cabeza y el cuello. Rev Chil Cir 1990; 42: 367-70.
15. Marín J, Montero M, Acuña J, Gallardo S, Guerra F. Extirpación tumor de cuerpo carotídeo y reemplazo de carótida interna con vena safena. Rev Chil Cir 1996; 48: 626-30.

16. Nazar G, Cabezas L, Godoy JM, Goycoolea M, Selman JM, Ortiz OA et al. Paragangliomas de Cabeza y Cuello. Rev Otorrinolaringol Cir Cabeza y Cuello 2005; 65: 203-14.

17. Baysal BE. Paragangliomas of the head and the neck. Otolaryngol Clin North Am 2001; 34: 86379.

18. Drovdlic CM, Myers en, Peters Ja, Baysal Be, Brackmann DE Slattery WH $3^{\text {RD }}$, Rubinstein WS. Proportion of heritable paraganglioma cases and associated clinical characteristics. Laryngoscope 2001; 111: 1822-7.

19. van der Mey AG, Jansen JC, van BaAlen. Management of carotid body tumors. Otolaryngol Clin North Am 2001; 34: 907-24.

20. Davidovic LB, Djukic VB, Vasic D, Sindjelic RP, Duvnjak SN. Diagnosis and treatment of carotid body paraganglioma: 21 years of experience at a clinical center of Serbia. World J Surg Oncol 2005; 3: 10.

21. Worsey MJ, Laborde AL, Bower T, Miller E, KRESOWIK TF. A evaluation of color duplex scanning in the primary diagnosis and management of carotid body tumors. Ann Vasc Surg 1992; 6: 904.

22. Hallett JW Jr, Nora JD, Hollier lH, Cherry KJ Jr, PaIrolero PC. Trends in neurovascular complications of surgical management for carotid body and cervical paragangliomas: A 50-year experience with 153 tumors. J Vasc Surg 1988; 7: 284-91.

23. Kafie FE, Freischlag JA. Carotid Body Tumors: The Role of Preoperative Embolization. Ann Vasc Surg 2001; 15: 237-42.

24. Smith JJ, Passman Ma, Dattilo JB, Guzmán RJ, Naslund TC, Netterville Jl. Carotid Body Tumor Resection: Does the Need for Vascular Reconstruction Worsen Outcome? Ann Vasc Surg 2006; 20: 435-9.

25. Pandya SK, Nagpal RD, Desai AP, Purohit at. Death following external carotid arterial embolization for a functioning glomus jugular chemodectoma. J Neurosurg 1978; 48: 1030. 